Ejections of Magnetic Structures Above a Spherical Wedge Driven by a Convective Dynamo with Differential Rotation

\title{
Warnecke, Jörn
}

$2012-10$

Warnecke , J , Käpylä , P J , Mantere , M J \& Brandenburg , A 2012 , ' Ejections of Magnetic Structures Above a Spherical Wedge Driven by a Convective Dynamo with Differential Rotation ' , Solar Physics , vol. 280 , no. 2 , pp. 299-319 . https://doi.org/10.1007/s11207-012-0108-4

http://hdl.handle.net/10138/39181

https://doi.org/10.1007/s11207-012-0108-4

acceptedVersion

Downloaded from Helda, University of Helsinki institutional repository.

This is an electronic reprint of the original article.

This reprint may differ from the original in pagination and typographic detail.

Please cite the original version. 


\title{
Coronal ejections driven by a convective dynamo with differential rotation in a sphere
}

\author{
Jörn Warnecke ${ }^{1,2}$ • Petri J. Käpylä ${ }^{1,3}$. \\ Maarit J. Mantere ${ }^{3}$. Axel Brandenburg ${ }^{1,2}$
}

(C) Springer $\bullet \bullet \bullet \bullet$

\begin{abstract}
We combine a convectively driven dynamo in a spherical shell with an isothermal density-stratified exterior that mimics a stellar corona to study the emergence and ejections of magnetic field structures. This approach is an extension of earlier models where we employed forced turbulence simulations to generate magnetic fields. A spherical wedge is used which consists of a convection zone and a corona up to more than twice the radius of the sphere. The wedge contains a quarter of the azimuthal extent of the sphere and $150^{\circ}$ in latitude. The magnetic field is self-consistently generated by the turbulent motions due to convection underneath the surface. Magnetic fields are found to emerge at the surface and are ejected to the coronal part of the domain. These ejections occur in irregular intervals and are smaller than in earlier work. We associate these events with coronal mass ejections on the Sun.
\end{abstract}

Keywords: Magnetic fields, Corona; Coronal Mass Ejections, Theory; Interior, Convective Zone; Turbulence; Helicity, Current

\section{Introduction}

Recent observations of the Solar Dynamic Observer (SDO) have provided us with a record of impressive solar eruptions. These eruptions are mostly associated with coronal mass ejections (CMEs). These are events through which the Sun sheds hot plasma from the corona into the interplanetary space. The energy causing such huge eruptions is stored in the magnetic field and can be released due to reconnection of field lines (Sturrock, 1980; Antiochos et al., 1999). A fraction of CMEs are directed towards the Earth, hitting its magnetosphere and causing phenomena like aurorae. Furthermore, encounters with CMEs can cause sudden outages of GPS signals due to ionospheric scintillation. The resulting radiation

\footnotetext{
1 Nordita, AlbaNova University Center, Roslagstullsbacken 23, SE-10691 Stockholm, Sweden, email: joern@nordita.org 2 Department of Astronomy, Stockholm University, SE-10691 Stockholm, Sweden

3 Department of Physics, Gustaf Hällströmin katu 2a (PO BOX 64), FI-00014 Helsinki University, Finland
} 
dose from such events poses risks to astronauts. This is now also of concern to airlines, because the radiation load during polar flights can reach annual limits, especially for pregnant women. This leads to a great interest of scientists in many fields.

However, there is an additional motivation which comes along with the space weather effects. The solar dynamo, which is broadly believed to be responsible for the generation of the solar magnetic field, needs to be sustained by shedding magnetic helicity from the Sun's interior, as pointed out in Blackman and Brandenburg (2003). Mean-field and direct numerical simulations have shown that the magnetic field generation is catastrophically quenched at high magnetic Reynolds numbers in closed systems that do not allow magnetic helicity fluxes Vainshtein and Cattaneo, 1992; Brandenburg and Subramanian, 2005). The magnetic Reynolds number, which is the ratio of the advective to the diffusive term in the induction equation, is known to be very large in the Sun, therefore implying catastrophic quenching of the solar dynamo unless efficient magnetic helicity fluxes occur. In the work by Blackman and Brandenburg (2003) these fluxes can be provided by the eruptive events like CMEs. Observations, see Plunkett et al. (2000) and Régnier et al. (2002), and a recent study by Thompson et al. (2011), where the observations are compared with numerical models, suggest that CMEs have a twisted magnetic structure, implying that CMEs transport helicity outwards.

There has been significant progress in the study of CMEs in recent years. In addition to improved observations from spacecrafts like SDO or the Solar TErestical RElation Observatory (STEREO), there have also been major advances in the field of numerical modeling of CME events (Russev et al., 2003; Antiochos et al., 2009). However, the formation and the origin of eruptive events like CMEs is not yet completely understood. Simulating CMEs and their formation is challenging. Leaving the difficulties of modeling the interstellar space aside, a CME, after being ejected in the chromosphere or lower corona, travels over a larger radial distance to the upper corona. In this environment the density and the temperature vary several orders of magnitude, which is not easy to handle in numerical models. Additionally, the origin of the CMEs is assumed to relate to the magnetic fields and the velocity pattern at the surface. However, the surface magnetic and velocity fields are strongly rooted in the solar convection zone, where convective motions, in interplay with differential rotation, generate the magnetic field and the velocity patterns that are observed at the surface. The majority of the researchers modeling CMEs do not include the convection zone in their setup, and thereby neglect the effect of the magnetic and velocity fields being rooted to this layer. Most often the initial conditions for the magnetic and velocity fields are prescribed or taken from 2D observations, see for example Antiochos et al. (1999) and Török and Kliem (2003).

Another approach is to study the emergence of flux ropes from the lower convection zone into the corona. There the convective motions follow a selfconsistent model, but the flux ropes are prescribed and their origin is left unexplained. In the work of Fang et al. (2010) and Martnez-Sykora et al. (2008) the focus lies on the emergence of magnetic flux and the resulting features in the solar atmosphere; eruptive events have not been investigated with this setup. In earlier work (Warnecke and Brandenburg, 2010; Warnecke et al., 2011) we 
have developed a different approach. We combine the solar convection zone with a simple model of the solar corona. The magnetic field, which is generated by dynamo action underneath the solar surface, emerges through the surface and is ejected out of the domain. Our focus lies on the connection of the dynamo-generated field and eruptive events like CMEs. Therefore, we first used a simplified coronal model and drove the dynamo with forced turbulence. These simplifications allowed us to study the emergence and a new mechanism to drive ejections in great detail. In Warnecke et al. (2011), we improved the setup of Warnecke and Brandenburg (2010) by using a spherical coordinate system and helical forcing with opposite sign in each hemisphere to mimic the effects of differential rotation. In addition, we applied a stratification due to gravity in an isothermal fluid. To improve this model, we now employ convection to generate the velocity field. The turbulent motions driving the generation of magnetic field are now self-consistently generated by convective cells, operating underneath the surface. The setup of the convection zone follows ideas of Käpvlä et al. (2008, 2010, 2011a,b) with an extended cooling layer to mimic a solar corona. The results of this work fit well with the results obtained by earlier work and observations. There are other approaches simulating convection in massive hot stars, which have thin subsurface convection zones Cantiello et al. (2011a,b).

The remainder of the paper is organized as follows: the numerical model is described in Section 2, and the results and conclusions are presented in Sections 3 and 4 , respectively.

\section{The model}

As in Warnecke and Brandenburg (2010) and Warnecke et al. (2011) a two-layer model is used, which combines the convection zone and the corona in a single domain. Our convection zone is similar to that in Käpylä et al. (2010, 2011b). The domain is a segment of the Sun and is described in spherical polar coordinates $(r, \theta, \phi)$. We model the convection zone starting at radius $r=0.7 R$ and the solar corona until $r=R_{c}$, where $R c=1.4 R$ and in some runs $R_{c} \approx 2 R$, where $R$ is the solar radius, used from here on as our unit length. In the latitudinal direction, our domain extends from colatitude $\theta=15^{\circ}$ to $165^{\circ}$ and in the azimuthal direction from $\phi=0^{\circ}$ to $90^{\circ}$. We solve the following equations of compressible magnetohydrodynamics,

$$
\begin{aligned}
\frac{\partial \boldsymbol{A}}{\partial t} & =\boldsymbol{U} \times \boldsymbol{B}+\eta \boldsymbol{\nabla}^{2} \boldsymbol{A}, \\
\frac{\mathrm{D} \ln \rho}{\mathrm{D} t} & =-\boldsymbol{\nabla} \cdot \boldsymbol{U}, \\
\frac{\mathrm{D} \boldsymbol{U}}{\mathrm{D} t} & =\boldsymbol{g}-2 \boldsymbol{\Omega} \times \boldsymbol{U}+\frac{1}{\rho}(\boldsymbol{J} \times \boldsymbol{B}-\boldsymbol{\nabla} p+\boldsymbol{\nabla} \cdot 2 \nu \rho \mathbf{S})-\boldsymbol{D}(r, \theta), \\
T \frac{\mathrm{D} s}{\mathrm{D} t} & =\frac{1}{\rho} \boldsymbol{\nabla} \cdot K \boldsymbol{\nabla} T+2 \nu \mathbf{S}^{2}+\frac{\mu_{0} \eta}{\rho} \boldsymbol{J}^{2}-\Gamma_{\mathrm{cool}},
\end{aligned}
$$

where the magnetic field is given by $\boldsymbol{B}=\boldsymbol{\nabla} \times \boldsymbol{A}$ and thus obeys $\boldsymbol{\nabla} \cdot \boldsymbol{B}=0$ at all times. The vacuum permeability is given by $\mu_{0}$, whereas magnetic diffusivity 
and kinematic viscosity are given by $\eta$ and $\nu$, respectively. $\mathrm{D} / \mathrm{D} t=\partial / \partial t+\boldsymbol{U} \cdot \boldsymbol{\nabla}$ is the advective time derivative, $\rho$ is the density, and $\boldsymbol{U}$ is the velocity. The traceless rate-of-strain tensor is given by

$$
\mathrm{S}_{i j}=\frac{1}{2}\left(U_{i ; j}+U_{j ; i}\right)-\frac{1}{3} \delta_{i j} \boldsymbol{\nabla} \cdot \boldsymbol{U},
$$

where semicolons denote covariant differentiation; see Mitra et al. (2009) for details. $\boldsymbol{\Omega}=\Omega_{0}(\cos \theta,-\sin \theta, 0)$ is the rotation vector, $p$ is the pressure, and $K$ is the radiative heat conductivity. $D(r, \theta)$ describes damping in the corona, see Section 2.2 for details. The gravitational acceleration is given by

$$
\boldsymbol{g}=-G M \boldsymbol{r} / r^{3},
$$

where $G$ is the gravitational constant or Newton constant, and $M$ is the mass of the star or the Sun. The fluid obeys the ideal gas law following $p=(\gamma-1) \rho e$, where $\gamma=c_{p} / c_{v}=5 / 3$ is the ratio of specific heats at constant pressure and volume, respectively, and $e=c_{v} T$ is the internal energy density.

\subsection{Initial setup and boundary conditions}

For the thermal stratification in the convection zone, we consider a simple analytical setup instead of profiles from solar structure models as in e.g. Brun et al. (2004). The hydrodynamic temperature gradient is given by

$$
\frac{\partial T}{\partial r}=\frac{-|\boldsymbol{g}|}{c_{v}(\gamma-1)(m+1)},
$$

where $m=m(r)$ is the radially varying polytropic index. We use Equation (7) as the lower boundary condition for the temperature. This gives the logarithmic temperature gradient $\nabla$ (not to be confused with the operator $\nabla$ ) as:

$$
\nabla=\frac{\partial \ln T}{\partial \ln p}=\frac{1}{m+1} .
$$

The stratification is convectively unstable if $\nabla-\nabla_{\text {ad }}>0$, where $\nabla_{\text {ad }}=1-1 / \gamma$ is the adiabatic temperature gradient, corresponding to $m<1.5$. We choose $m=1$ in the convectively unstable layer underneath the surface $r<R$. The region above $r=R$ is stably stratified and isothermal due to a cooling term $\Gamma_{\text {cool }}$ in the entropy equation. The $\Gamma_{\text {cool }}$ term is $r$-dependent and causes a smooth transition to the isothermal layer representing the corona. The density stratification is obtained by requiring the hydrostatic equilibrium condition to be satisfied.

The thermal conductivity follows from the constancy of the luminosity $L$ throughout the domain and is given by

$$
K=\frac{L}{4 \pi r^{2} \partial T / \partial r} .
$$

In order to speed up the thermal relaxation processes, we apply shallower profiles, corresponding to $\rho \propto T^{1.4}$, for the thermal variables within the convectively 


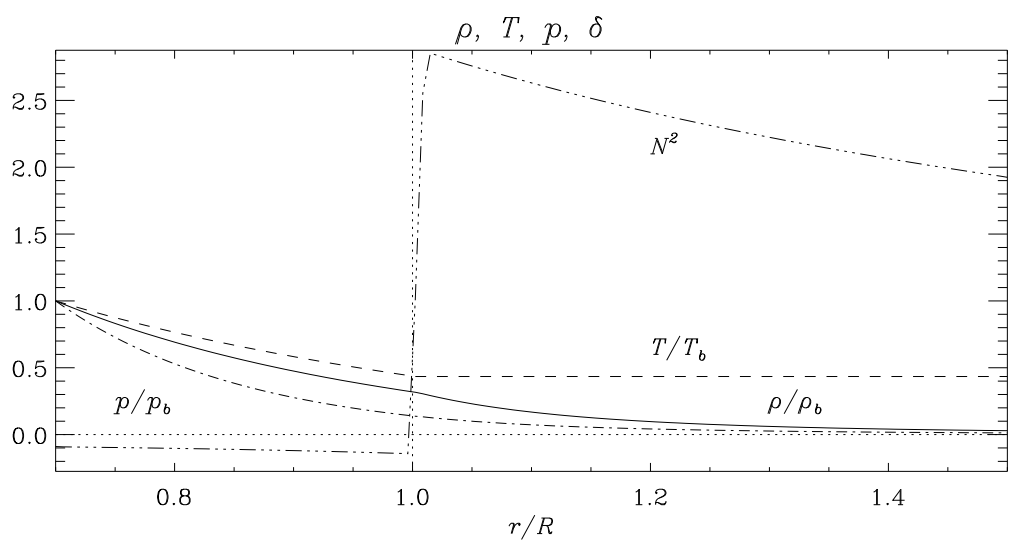

Figure 1. Initial stratification of temperature (solid line), density (dashed), pressure (dotdashed) and the Brunt-Väisälä frequency $N^{2}=-\left(|\boldsymbol{g}| / H_{p}\right)\left(\nabla-\nabla_{\mathrm{ad}}\right)$ (dash-triple-dotted) for Run A5. The subscripts $b$ refers to the values at $r=0.7 R$. The dotted horizontal (vertical) line denotes the value of zero $(r=R)$.

unstable layer. The value $m=1$ is just used in the convection zone to determine the thermal conductivity. In Figure 1 we show the initial non-convecting stratification. The temperature gradient at the bottom of the domain is set to a constant value, which leads to a constant heat flux into the domain. In the corona the gradient is smoothly set to 0 by using a $r$ dependent cooling function $\Gamma_{\text {cool }}$, which is added to the entropy evolution Equation (4). The cooling term is given by

$$
\Gamma_{\text {cool }}=\Gamma_{0} f(r)\left(\frac{c_{\mathrm{s}}^{2}-c_{s 0}^{2}}{c_{s 0}^{2}}\right),
$$

where $f(r)$ is a profile function equal to unity in $r>R$ and smoothly connecting to zero in $r \leq R$, and $\Gamma_{0}$ is a cooling luminosity chosen so that the sound speed in the corona relaxes towards $c_{\mathrm{s} 0}^{2}=c_{\mathrm{s}}^{2}\left(r=R_{c}\right)$. Whether the stratification is convectively unstable can be determined by the Brunt-Väisälä frequency:

$$
N^{2}=|\boldsymbol{g}|\left(\frac{1}{\gamma} \frac{\partial \ln p}{\partial r}-\frac{\partial \ln \rho}{\partial r}\right)=-\frac{|\boldsymbol{g}|}{H_{p}}\left(\nabla-\nabla_{\mathrm{ad}}\right),
$$

where $H_{p}=\partial r / \partial \ln p$ is the pressure scale height. If the frequency is negative, the stratification is unstable.

We apply periodic boundary conditions in the azimuthal direction. For the velocity we apply stress-free boundary conditions at all other boundaries. The temperature gradient is imposed at the bottom and constant temperature is imposed at the top. Thermodynamic variables have zero gradients at the latitude boundaries. We use perfect conductor boundaries for the magnetic field at the latitudinal and at the lower radial boundaries, and vertical field conditions at the outer radial boundary. The latter is due to the fact that the solar wind pushes 
the magnetic field to open field lines and at a solar radius of $r=1.5 R$ till $r=2 R$ the field lines are mostly vertical (Levine et al., 1982).

We use the PEncil Cod 1 , which uses sixth-order centered finite differences in space and a third-order accurate Runge-Kutta scheme in time; see Mitra et al. (2009) for the extension of the PENCIL CODE to spherical coordinates. We use a grid size of $128 \times 128 \times 64$ mesh points (Runs A2, A3, A4, A5, Ar1, C1) ,256 × $256 \times 128$ (Runs A12a, A4a, A5a, A5b, A5c, Ar1a, Ar2a, E1a, F1, F1a, F1b) and $256 \times 128 \times 64$ (Run H1).

\subsection{Velocity damping in the corona}

Whether the solar corona rotates like a solid body or differentially coupled with the photosphere is currently unclear. In recent work by Wöhl et al. (2010), where SOHO-EIT data of the bright points in the solar corona are used to estimate the rotation speeds, it was found that the corona rotates similarly as the small magnetic features in the photosphere. However, the observations of the "boot" coronal hole by SKYLAB suggested rigid rotation (Timothy et al., 1975). Due to the low plasma $\beta$ (defined as the ratio of the thermal to magnetic pressure $\left.\beta=2 \mu_{0} p / \boldsymbol{B}^{2}\right)$ in the solar corona, the fluid motions are dominated by the magnetic fields whose footpoints are anchored in the photosphere or even further down. So the magnetic field then might be rigid enough to prevent differential rotation of the solar corona. However, the observed bright points and other features in the corona are strongly correlated with the magnetic field so they can give a misleading picture about the global rotation of the corona.

In our simulations, the Coriolis force is included in the momentum equation as a consequence of the rotation. In the solar corona the density is more than 14 orders of magnitude smaller than in the lower convection zone. Because of the weak density stratification in our simulation, the Coriolis force in our corona is too strong and can cause non-coronal effects like magnetorotational instability. To avoid this - at least for runs with rapid rotation - we apply a damping function $\boldsymbol{D}(r, \theta)$ in the momentum equation, which is given by

$$
\boldsymbol{D}(r, \theta)=\frac{1}{\tau} D_{0}(r-R) \overline{\boldsymbol{U}}(r, \theta),
$$

where

$$
D_{0}(r-R)=\frac{1}{2}\left[\tanh \left(\frac{r-R}{w}\right)+1\right],
$$

with $\tau$ being the damping time and $w$ the width of the transition layer from convection zone to the corona, and where the overbar denotes $\phi$ averaging.

2.3. Units, nondimensional quantities, and parameters

Dimensionless quantities are obtained by setting

$$
G M=\rho_{\mathrm{b}}=p_{\mathrm{b}}=c_{p}=\mu_{0}=1,
$$

\footnotetext{
${ }^{1}$ http://pencil-code.googlecode.com
} 
where $\rho_{\mathrm{b}}$ is the density at $r=0.7 R$, and $p_{\mathrm{b}}$ is the reference value of the pressure. Below, we will describe the properties of the runs by the following dimensionless parameters: fluid Reynolds number $\operatorname{Re}=u_{\mathrm{rms}} / \nu k_{\mathrm{f}}$, magnetic Reynolds number $\operatorname{Re}_{M}=u_{\mathrm{rms}} / \eta k_{\mathrm{f}}$ where $k_{\mathrm{f}} R=21$ is the wavenumber of the energy-carrying eddies and $u_{\mathrm{rms}}$ is the volume averaged rms velocity for the convection zone $(r \leq R)$. The magnetic Prandtl number $\operatorname{Pr}_{M}=\nu / \eta=\operatorname{Re}_{M} /$ Re and the Coriolis number $\mathrm{Co}=2 \Omega_{0} / u_{\mathrm{rms}} k_{\mathrm{f}}$. In the following analysis, we use $\phi$ averages, defined as $\bar{F}(r, \theta, t)=\int F(r, \theta, \phi, t) \mathrm{d} \phi / 2 \pi$. Occasionally we also use time averages denoted by $\langle.\rangle_{t}$. Time is expressed in units of $\tau=\left(u_{\mathrm{rms}} k_{\mathrm{f}}\right)^{-1}$, which is the eddy turnover time in the convection zone. We measure the magnetic field strength as the rms value averaged over the convection zone $B_{\text {rms }}$, where we often normalize this value with the equipartition value of the magnetic field defined by $B_{\mathrm{eq}}^{2}=$ $\mu_{0}\left(\bar{\rho} u_{\mathrm{rms}}^{2}\right)_{r \leq R}$. The relative kinetic helicity $h_{\mathrm{rel}}(r, t)=\overline{\boldsymbol{\omega} \cdot \boldsymbol{u}} / \omega_{\mathrm{rms}} u_{\mathrm{rms}}$, where $\boldsymbol{\omega}=\boldsymbol{\nabla} \times \boldsymbol{u}$ is the vorticity and $\omega_{\text {rms }}$ the rms value averaged over the convection zone.

\section{Results}

\subsection{Hydrodynamic phase of the simulations}

After around 100 turnover times, the convection has reached saturation and we find convection cells as typical patterns in the radial velocity just below the surface. The size of these cells depends strongly on the strength of rotation and the degree of density stratification; see also Käpylä et al. (2011b). In addition, extremely low Reynolds numbers also prevents smaller cells to form. As an example, we plot the radial velocity $U_{r}$ at $r=0.89 R$ for Runs A5, A5a and Ar1 in Figure 2, The Run A5 has a low fluid Reynolds number and therefore the convection cells are large, see Table 1. The flow pattern shows clear 'banana cells' as in previous work with comparable Coriolis parameter, cf. Käpvlä et al. (2011a). A higher fluid Reynolds number and higher resolution, as in Run A5a, allow the velocity field to form more complex structures. However, the banana cells are still visible. If one now looks at a simulation with more rapid rotation (Run Ar1 plotted in the right-most panel of Figure 2) with a Coriolis number of $\mathrm{Co}=50$, the number of banana cells increases and they are more clearly visible than in Run A5a.

In the Sun, differential rotation is an important element to produce the magnetic field structures observed at large scales, exhibiting a cyclic behavior over time, as manifested by the sunspot cycle. To illustrate the differential rotation profiles generated in the simulations, we plot the azimuthally averaged angular velocity, $\bar{\Omega}=\bar{U}_{\phi} /(r \sin \theta)+\Omega_{0}$, for Runs A5, A5a, and Ar1 in the saturated state of the simulation, see Figure 3. In the plot, we show isocontours of angular velocity with solid black lines. The corona seems to rotate as a solid body outside the tangent cylinder whereas at higher latitude some differential rotation occurs also in the corona. In the three runs shown in Figure 3 the stratification in the whole domain is just $\rho_{\mathrm{b}} / \rho_{\mathrm{t}}=40$, which is extremely small if compared to the stratification of the Sun $\left(\rho_{\mathrm{b}} / \rho_{\mathrm{t}} \sim 10^{14}\right)$. Therefore, the Coriolis force 
Table 1. Summary of the runs. Re is the fluid Reynolds number, $\operatorname{Pr}_{M}$ is the magnetic Prandtl number, Co is the Coriolis number, and $h_{\text {rel }}$ is the maximum value of the relative kinetic helicity over each hemisphere, as defined in Section $2.3 \frac{\rho_{\mathrm{b}}}{\rho_{s}}$ and $\frac{\rho_{\mathrm{b}}}{\rho_{\mathrm{t}}}$ give the density ratios of the bottom of the convection zone and the surface and the top of the domain, respectively. $R_{c}$ indicates the top of the domain in the radial direction. In the right-most column we denote if damping for velocity in the corona is used $(\mathrm{Y})$ or not $(\mathrm{N})$, see Section 2.2

\begin{tabular}{lrccccccccc} 
Run & Resolution & $\operatorname{Re}$ & $\operatorname{Pr}_{M}$ & $\frac{B_{\mathrm{rms}}^{2}}{B_{\text {eq }}^{2}}$ & $\frac{\rho_{\mathrm{b}}}{\rho_{s}}$ & $\frac{\rho_{\mathrm{b}}}{\rho_{t}}$ & Co & $h_{\text {rel }}$ & $R_{c} / R$ & $D$ \\
\hline A2 & $128^{2} \times 64$ & 53 & 1 & 0.08 & 3.6 & 39 & 2.0 & 0.3 & 1.5 & $\mathrm{~N}$ \\
A2a & $265^{2} \times 128$ & 121 & 1 & 0.06 & 3.6 & 39 & 1.9 & 0.2 & 1.5 & $\mathrm{Y}$ \\
A3 & $128^{2} \times 64$ & 87 & 1 & $0.1-0.4$ & 3.6 & 39 & 5.2 & 0.30 & 1.5 & $\mathrm{~N}$ \\
A4 & $128^{2} \times 64$ & 14 & 2 & 1.9 & 3.6 & 39 & 16 & 0.3 & 1.5 & $\mathrm{~N}$ \\
A4a & $265^{2} \times 128$ & 67 & 1 & 0.30 & 3.6 & 39 & 14 & 0.2 & 1.5 & $\mathrm{~N}$ \\
A5 & $128^{2} \times 64$ & 3.2 & 10 & $0.1-0.4$ & 3.6 & 39 & 7 & 0.5 & 1.5 & $\mathrm{~N}$ \\
A5a & $265^{2} \times 128$ & 100 & 1 & 0.2 & 3.6 & 39 & 4.5 & 0.3 & 1.5 & $\mathrm{~N}$ \\
A5b & $265^{2} \times 128$ & 45 & 1 & 0.8 & 3.7 & 154 & 5.0 & 0.3 & 2.1 & $\mathrm{~N}$ \\
A5c & $265^{2} \times 128$ & 3.1 & 10 & $0.2-0.6$ & 3.6 & 39 & 7 & 0.4 & 1.5 & $\mathrm{~N}$ \\
Ar1 & $128^{2} \times 64$ & 38 & 1 & $2.0-6.0$ & 3.6 & 39 & 50 & 0.3 & 1.5 & $\mathrm{Y}$ \\
Ar1a & $265^{2} \times 128$ & 53 & 1 & 0.4 & 3.5 & 38 & 34 & 0.2 & 1.5 & $\mathrm{Y}$ \\
Ar2 & $128^{2} \times 64$ & 36 & 1 & $0.3-1.3$ & 3.6 & 63 & 37 & 0.05 & 1.5 & $\mathrm{Y}$ \\
Ar2a & $265^{2} \times 128$ & 88 & 1 & 0.01 & 3.5 & 38 & 52 & 0.15 & 1.5 & $\mathrm{Y}$ \\
C1 & $128^{2} \times 64$ & 8.0 & 1.5 & $0.2-0.5$ & 5.3 & 152 & 5.7 & 0.4 & 1.5 & $\mathrm{Y}$ \\
E1a & $265^{2} \times 128$ & 168 & 1 & 0.07 & 8.3 & 896 & 4.5 & 0.2 & 1.5 & $\mathrm{Y}$ \\
F1 & $265^{2} \times 128$ & 22 & 1.7 & 0.6 & 5.2 & 1732 & 60 & 0.2 & 2.1 & $\mathrm{Y}$ \\
F1a & $265^{2} \times 128$ & 29 & 1.5 & 0.02 & 5.3 & 1723 & 52 & 0.10 & 2.1 & $\mathrm{~N}$ \\
F1b & $265^{2} \times 128$ & 48 & 3 & 0.15 & 5.2 & 1743 & 6.4 & 0.25 & 2.1 & $\mathrm{~N}$ \\
H1 & $265^{2} \times 128$ & 25 & 4 & 0.29 & 5.8 & 1826 & 8.5 & 0.25 & 2.0 & $\mathrm{Y}$ \\
\hline
\end{tabular}
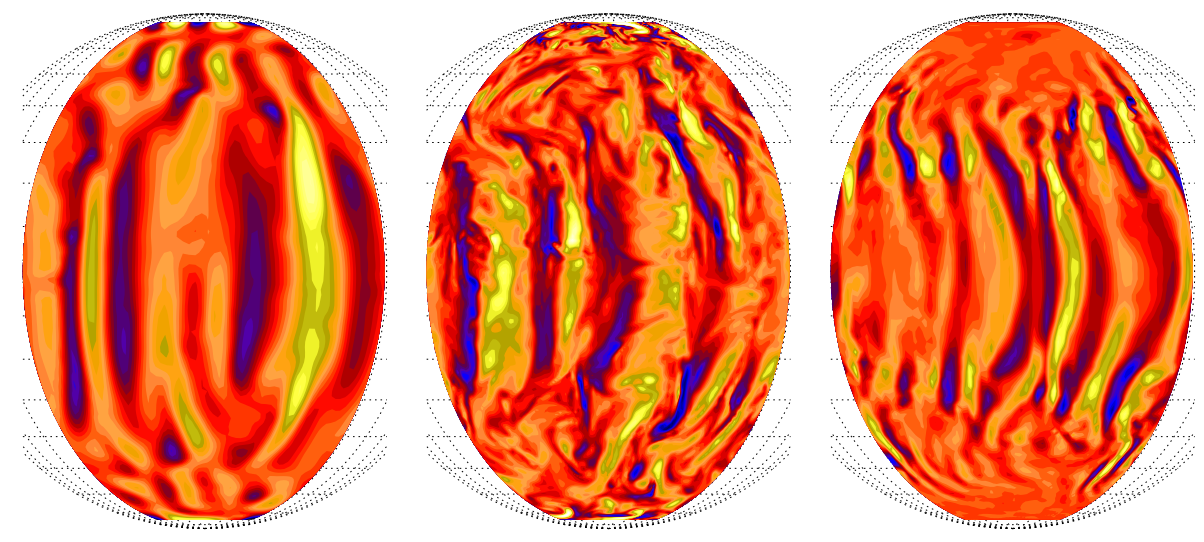

Figure 2. Radial velocity $\left(U_{r}\right)$ underneath the surface $(r=0.89 R)$ for Runs A5, A5a, Ar1. Dark blue shades represent negative and light yellow positive values. 

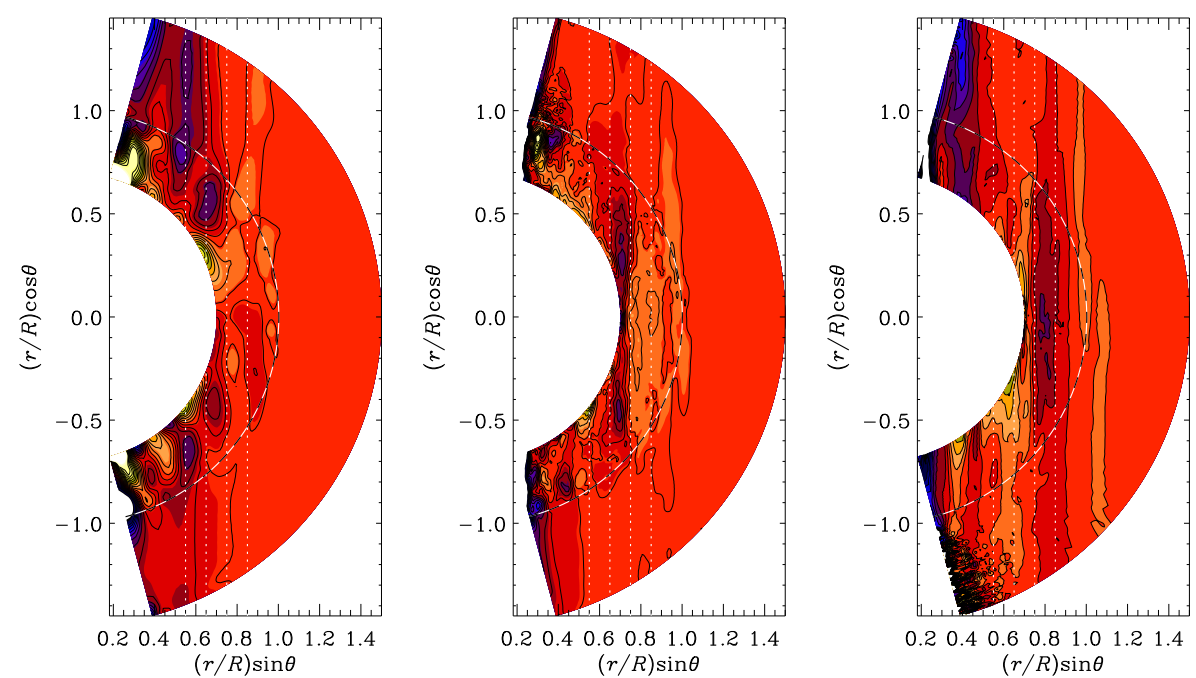

Figure 3. Differential rotation profiles $\bar{\Omega}(r, \theta)=\bar{U}_{\phi} /(r \sin \theta)+\Omega_{0}$ for Runs A5, A5a, Ar1. Dark blue shades represent low and light yellow high values, overplotted by the isocontours with solid black lines.

is acting much more strongly in the corona of our simulation than in reality. In the convection zone, we find quenching of convection due to rapid rotation. In Run A5, where the Coriolis number $\mathrm{Co}=7$, the lines of constant rotation rate are more radial than vertical and show super-rotation, i.e., the equator rotates faster than the poles. However, the convection cells are very big and have stronger local influence on $U_{\phi}$. Note that the rms velocity in Run A5 is two times smaller than in Run A5a, which has a higher resolution and higher fluid and magnetic Reynolds numbers $\left(\operatorname{Re}=\operatorname{Re}_{M}=100\right)$. Due to this, we find clear super-rotation, even though the Coriolis number is slightly lower $(\mathrm{Co}=4.5)$ than realized in Run A5. In the third case, Run Ar1, where the rotation is extremely rapid $(\mathrm{Co}=50)$, we also find super-rotation, where the lines of constant rotation rate are almost all vertical. In comparable work (Käpvlä et al., 2011a, b), superrotation has been found, when the Coriolis number was larger than 4 . This is similar to our results including the corona. In addition, there is a minimum of the rotation rate at mid-latitudes and a polar vortex at high latitudes. Solar rotation profiles, which show a comparable behavior have been found by several research groups (Miesch et al., 2000; Elliot et al., 2000; Käpvlä et al., 2011a,b). The region with the higher rotation rate near the equator is limited to upper convection and even penetrate the corona. In Run Ar1 the velocity damping described in Section 2.2 is used. By comparing the right-most panel of Figure 3 with damping to the left-most panels without it, it can be concluded that the damping does not make much of a difference to the coronal velocity structures.

Simulations with randomly forced turbulence (e.g. Warnecke and Brandenburg, 2010; Warnecke et al., 2011) have shown that the relative kinetic helicity $h_{\text {rel }}$ has a strong influence both on the generation of large-scale magnetic fields and the ejection events. In Warnecke and Brandenburg (2010) and Warnecke et al. 

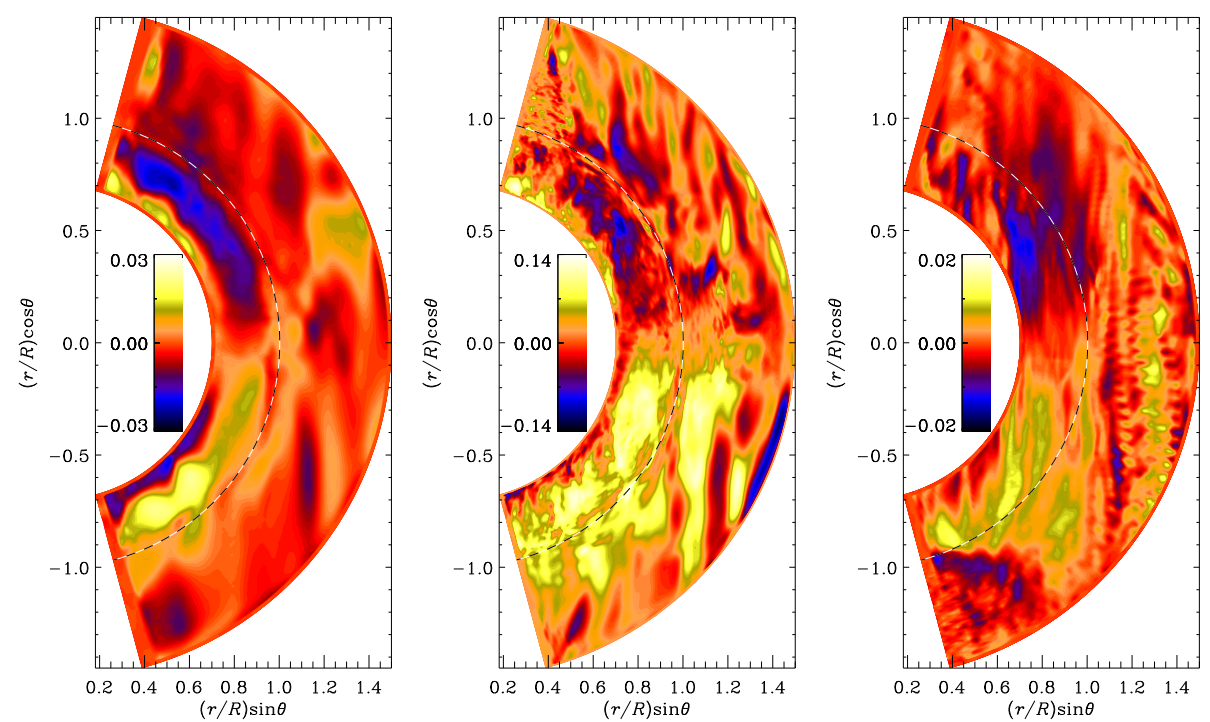

Figure 4. Relative helicity $h_{\mathrm{rel}}(r, t)=\overline{\boldsymbol{\omega} \cdot \boldsymbol{u}} / \omega_{\mathrm{rms}} u_{\mathrm{rms}}$ plotted for Runs A5, A5a, Ar1 Dark blue shades represent negative and light yellow positive values.

(2011) we has $h_{\text {rel }} \approx 1$, achieved by using a forcing function with purely helical plane waves. In the convection runs presented here, however, values of maximally $h_{\text {rel }}=0.5$ are obtained (for Run A5), at least at certain radii. In Figure 4 we present contour plots of azimuthally averaged relative helicity in the meridional plane for Runs A5, A5a and Ar1. All three show the typical sign rule of kinetic helicity under the influence of rotation, i.e. the northern hemisphere has a predominantly negative sign and in the southern a positive one. Very close to the bottom of the convection zone, the sign changes, which has earlier been reported by several authors both in Cartesian (e.g. Ossendrijver et al., 2001) and spherical geometries (e.g. Käpylä et al., 2010). Only in the Run Ar1 with rapid rotation, the behavior is not that clear. The relative helicity is no longer confined to the convection zone, but significant values occur also in the coronal region. The sign rule still holds within the convection zone, while a more complicated sign behavior is visible in the corona. The maximal values of the azimuthally averaged helicity are around $h_{\text {rel }}=0.3$, occurring close to the surface. In Run A5a, the maximum value is slightly higher and is located in the middle of the convection zone, although relatively high values are present in the corona as well. It is not yet completely clear how high values of relative kinetic helicity can be achieved; strong rotation tends to suppress it, whereas high stratification increases it. Also its exact role in generating coronal ejections is yet unclear.

\subsection{Convective dynamo}

The convective motions generate a large-scale magnetic field due to dynamo action. The magnetic field grows first exponentially and then suppresses the 

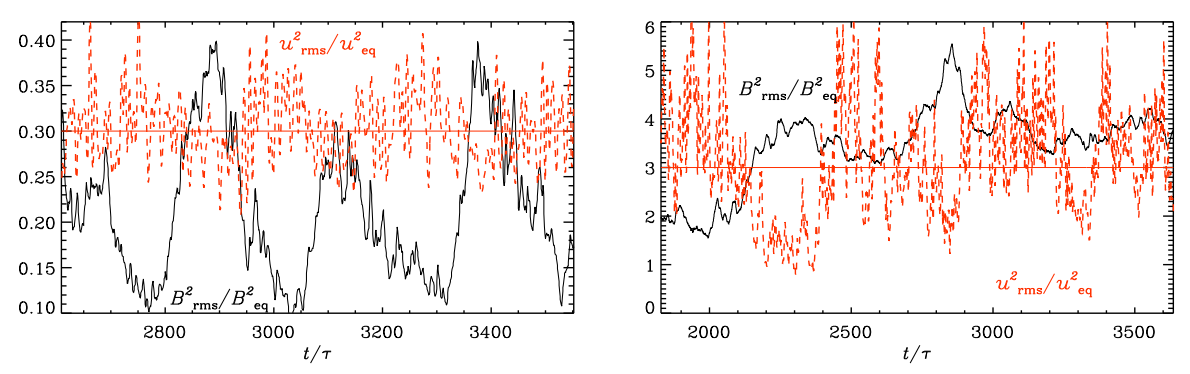

Figure 5. Phase relation of the magnetic field $\left(B_{\mathrm{rms}}^{2} / B_{\text {eq }}^{2}\right.$, solid black lines $)$ and the velocity field $\left(u_{\mathrm{rms}}(t)^{2} /\left\langle u_{\mathrm{rms}}^{2}\right\rangle_{t}\right.$, dashed red lines) in the convection zone for Runs A5 (left panel) and Ar1 (right). The velocity has been multiplied with a factor of 0.3 (left panel) and 3 (right), respectively, and smoothed over 5 neighboring data points. We indicate the time average value as a solid red line.

velocity field. The growth of the magnetic field saturates after around 200 to 1000 turnover times, depending on the Coriolis and Reynolds numbers. In the runs in Table 1, we obtain different dynamo solutions for the saturated field. In Run A5, for example, we find an oscillation of the volume-averaged rms magnetic field in the convection zone, see Figure 5 The growth tends to be sharper than the decline, the period being around $t / \tau=220$. The field reaches a maximum of $60 \%$ of the equipartition field strength, $B_{\text {eq }}$, which is comparable to the values obtained in the forced turbulence counterparts both in Cartesian and spherical coordinates (Warnecke and Brandenburg, 2010; Warnecke et al., 2011). Comparing this with the change of the kinetic energy, plotted as fluctuations of the rms velocity squared, we find an anti-correlation with respect to the magnetic field oscillation. The magnetic field is high (low), when the velocity is low (high). This behavior is not seen as clearly in the large-scale magnetic field which fluctuates in strength, but not in sign. As shown in Figure 6 for Run A5, the $\bar{B}_{\phi}$ and $\bar{B}_{r}$ have local maxima in time and in latitude, but the overall structure is nearly constant in time. Even though the large-scale field structure is stationary, the small-scale structures show an equatorward migration near the equator. The azimuthal velocity $\bar{U}_{\phi}$ over time and latitude is shown in Figure 8 shows minima at the same times as the maxima of the magnetic field occur. In Run A5a, the occurrence of strong magnetic fields suppresses the differential rotation. The pattern of the azimuthal velocity is symmetric about the equator and shows an oscillatory behavior, which is not that clear in the large-scale magnetic field.

In Run Ar1a, the magnetic field reaches up to 2.3 equipartition, but does not show a periodic oscillation, see Figure 5. In comparable work (Käpvlä et al., 2010), similar values for the field strength were found. However, the rms velocity is also quenched, when the magnetic field is high. Looking at the $\bar{B}_{\phi}$ and $\bar{B}_{r}$, plotted over time and latitude in Figure 7 the large-scale magnetic field is similar to Run A5, that is constant in time and does not show any oscillation. Comparing the two hemispheres, however, the field structure is antisymmetric. In the $\bar{U}_{\phi}$ plot in Figure 8, we find just one localized minimum, which coincides with the low values of $u_{\mathrm{rms}}(t)^{2} /\left\langle u_{\mathrm{rms}}^{2}\right\rangle_{t}$ between $t / \tau=2100$ and 2400 . 

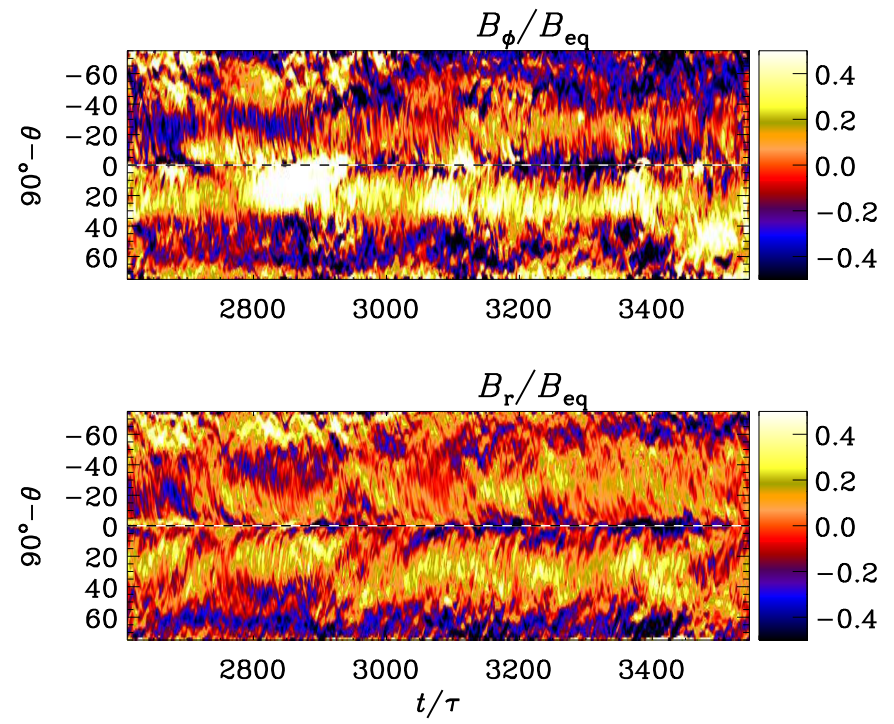

Figure 6. Variation of $\bar{B}_{\phi}$ and $\bar{B}_{r}$ in the convection zone at $r=0.9 R$ for Run Ar1. Dark blue shades represent negative and light yellow positive values. The dotted horizontal lines show the location of the equator at $\theta=\pi / 2$. The magnetic field is normalized by the equipartition value.
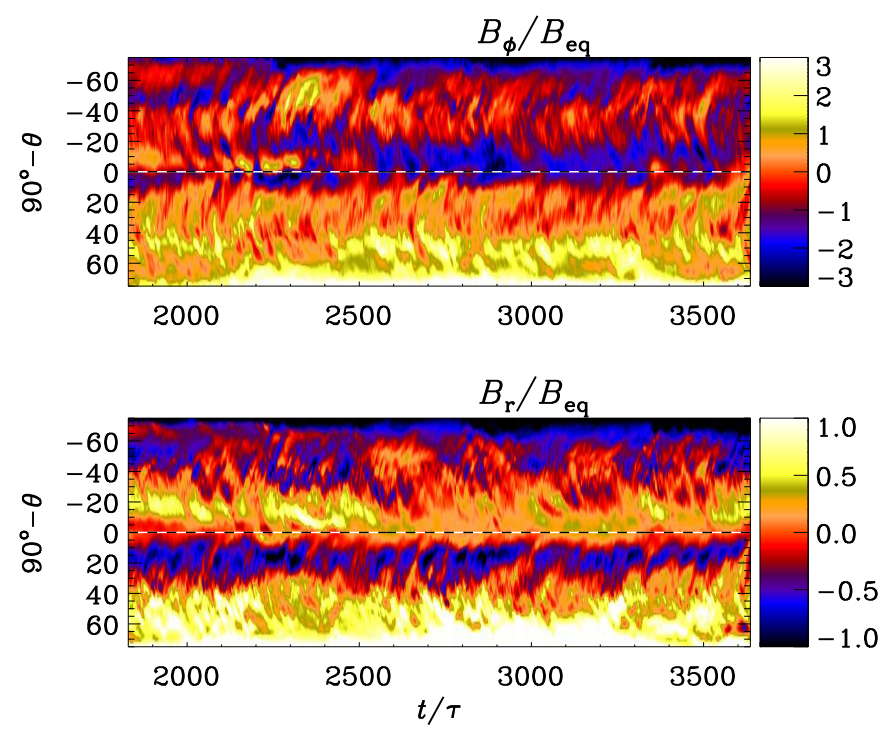

Figure 7. Variation of $\bar{B}_{\phi}$ and $\bar{B}_{r}$ in the convection zone at $r=0.9 R$ for Run Ar1. Dark blue shades represent negative and light yellow positive values. The dotted horizontal lines show the location of the equator at $\theta=\pi / 2$. The magnetic field is normalized by the equipartition value. 

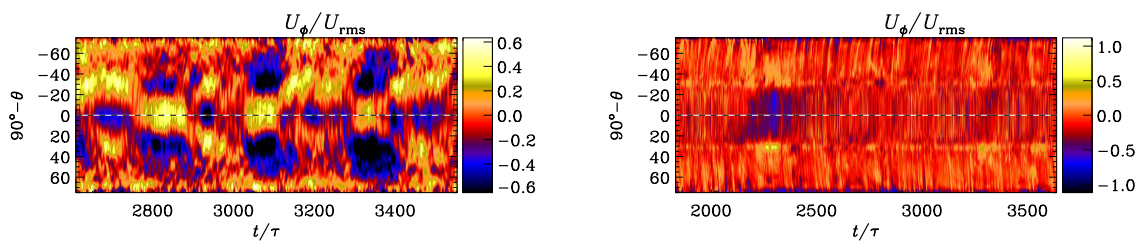

Figure 8. Variation of $\bar{U}_{\phi}$ in the convection zone at $r=0.8 R$ for Run A5 (left panel) and Run Ar1 (right panel). Dark blue shades represent negative and light yellow positive values. The dotted horizontal lines show the location of the equator at $\theta=\pi / 2$. The velocity is normalized by the mean rms velocity in the convection zone.
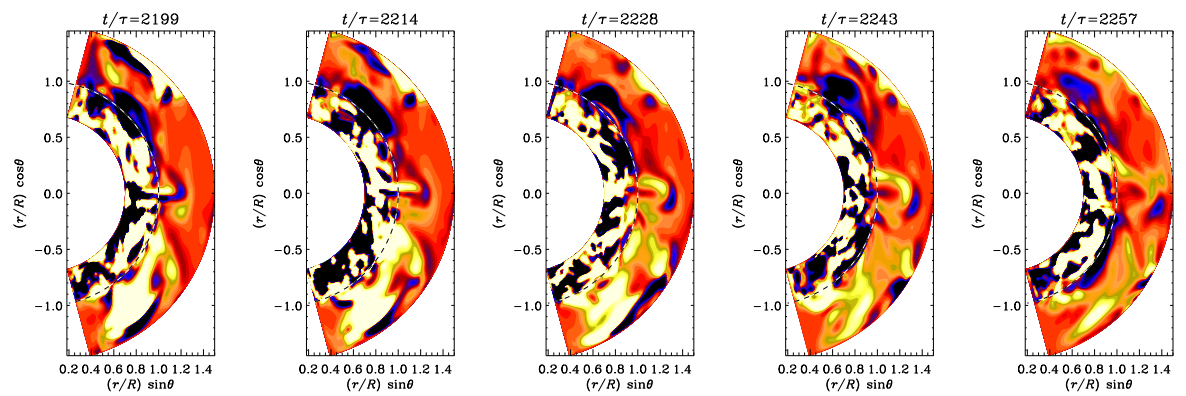

Figure 9. Time series of a coronal ejection near the equator $(\theta=\pi / 2)$. The normalized current helicity, $\mu_{0} R \overline{\boldsymbol{J} \cdot \boldsymbol{B}} /\left\langle\overline{\boldsymbol{B}^{2}}\right\rangle_{t}$, is shown in a color-scale representation from different times; dark blue represents negative and light yellow positive values. The dashed horizontal lines show the location of the surface at $r=R$. Taken from Run A5.

\subsection{Coronal ejections}

As seen in Table 1, 19 runs have been performed, which cover a considerable parameter space. However, only a small fraction of events can be identified with actual coronal ejections similar to the ones seen in Warnecke and Brandenburg (2010) and Warnecke et al. (2011). Especially the Runs A5 and Ar1a show some clear ejection events. There the magnetic field emerges out of the convection zone and is ejected as an isolated structure. In Figure 9 we have plotted the normalized current helicity, $\mu_{0} R \overline{\boldsymbol{J} \cdot \boldsymbol{B}} /\left\langle\overline{\boldsymbol{B}^{2}}\right\rangle_{t}$, as a time series for Run A5. At small scales, the current helicity density, $\boldsymbol{J} \cdot \boldsymbol{B}$, is a good proxy for magnetic helicity density, $\boldsymbol{A} \cdot \boldsymbol{B}$, and is, as opposed to this quantity, gauge invariant. In addition the current helicity can be an indicator of helical magnetic structures, which are believed to be present in coronal mass ejections (Plunkett et al., 2000; Régnier et al., 2002; Thompson et al., 2011). Close to the equator a bipolar structure emerges through the surface. The inner bulk has a positive current helicity in Figure 9 represented by a yellow color and pushes an arc with negative current helicity in its front. Such bipolar ejections have been identified in earlier work (Warnecke et al., 2011) and compared with the 'three-part structure' of coronal mass ejection which is described in Low (1996). The three parts consist 

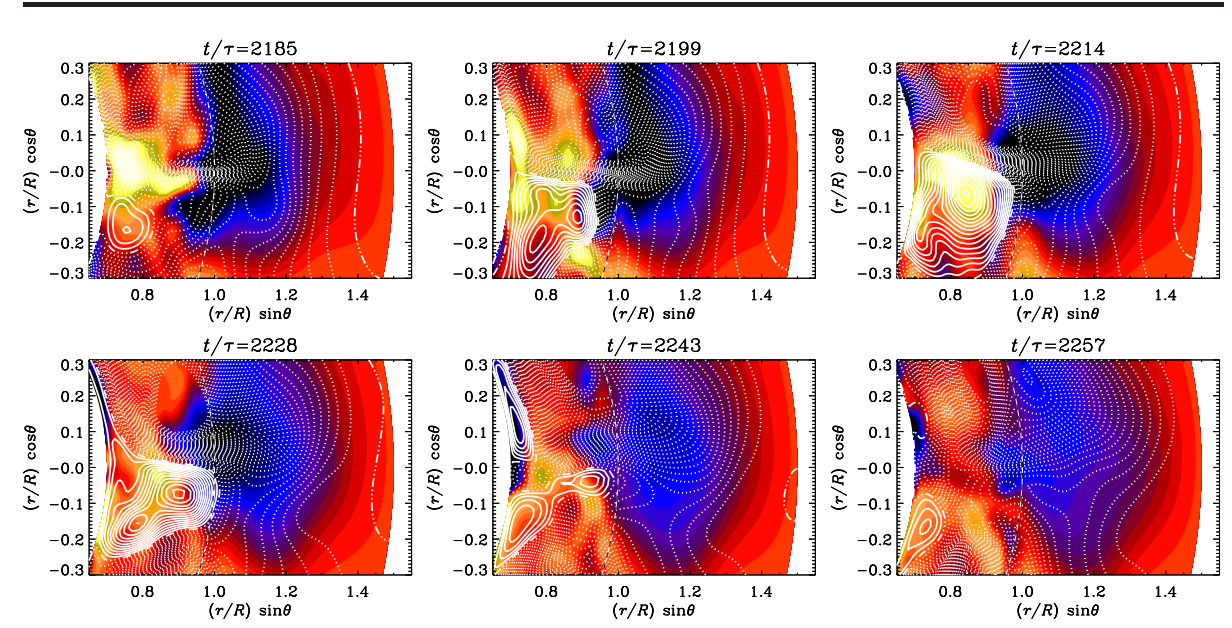

Figure 10. Time series of formation of a plasmoid ejections zoomed in the region of the ejection. Contours of $r \sin \theta \bar{A}_{\phi}$ are shown together with a color-scale representation of $\bar{B}_{\phi}$; dark blue stands for negative and light yellow for positive values. The contours of $r \sin \theta \bar{A}_{\phi}$ correspond to field lines of $\overline{\boldsymbol{B}}$ in the $r \theta$ plane, where solid lines represent clockwise magnetic field lines and the dashed ones counter-clockwise. The dashed horizontal lines show the location of the surface at $r=R$. Taken from Run A5.

out of a prominence, which is similar to the bulk in our simulations, a front with an arc shape corresponding to our arc and the cavity between these two features. Even though the domain of the simulation is larger in the $\theta$ direction as in Warnecke et al. (2011), the ejections are much smaller, which is actually closer to the CMEs observed on the Sun. The difference in size is mostly due to the more complex and fluctuating magnetic field in convection runs than in forced turbulent ones, see more in Section 4. In the sequence of images of Figure 9, an ejection near the equator reaches the outer boundary and leaves the domain. To investigate the mechanism driving the ejection, we look at the dynamics of the magnetic field in Figure 10, where field lines of the azimuthally averaged mean field are shown as contours of $r \sin \theta \bar{A}_{\phi}$ and colors represent $\bar{B}_{\phi}$ for the same time series. During the ejection, one can notice a strong concentration of magnetic field lines that are directed radially outwards. This concentration appears first underneath the surface and then emerges below the current helicity structure and follows it up into the corona. Investigating the direction of the magnetic field lines in the time series in Figure 10, an X-point can be found. In the first panel, at $r=1.07 R$ and $\theta=\pi / 2+0.1$, the magnetic field lines form a junction-like shape. The dotted line represents a counter-clockwise oriented field loop, so at the two corners of the junction there are field lines with opposite signs. After around 14 turnover times this "junction" has reconnected at the same position where the ejection is detected. It appears that these two events are related to each other.

The ejection causes also a strong variation in the density. If the time-averaged density profile is subtracted from instantaneous ones, the density fluctuations are obtained. After removing the density stratification one obtains $\Delta \overline{\rho(t)}=\overline{\rho(t)}-$ 


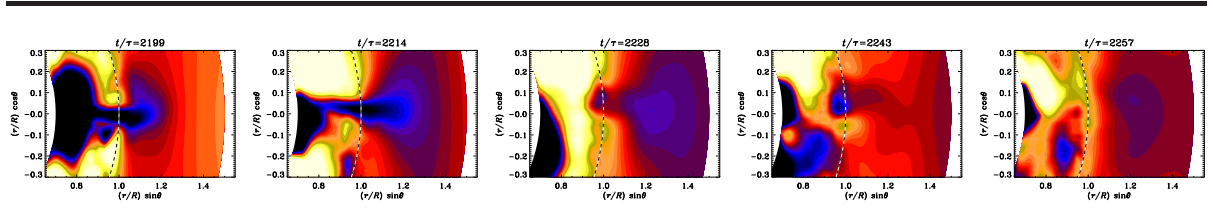

Figure 11. Time series of the evolution of the density fluctuations $\Delta \overline{\rho(t)}=\overline{\rho(t)}-\langle\bar{\rho}\rangle_{t}$ in the corona, zoomed in the region of the ejection. In the color-scale, dark blue represents negative and light yellow positive values. The dashed horizontal lines show the location of the surface at $r=R$. Taken from Run A5.

$\langle\bar{\rho}\rangle_{t}$. We plot these density fluctuations, $\Delta \overline{\rho(t)}$, for the same times in Figure 9 in Figure 11 to visualize the effect of the ejection on the density. The density in the ejection is much lower than in the rest of the corona. One interpretation could be that the strong magnetic field reduces the density to achieve total pressure equilibrium and the ejection rises then because of magnetic buoyancy. Such an effect is also seen by inspecting other ejections. In the solar corona, the plasma $\beta$ is very low because of the low density. There the magnetic field can drag dense plasma from the lower corona to its upper part. In our simulations the density stratification of the convection zone is much lower compared to the Sun. Therefore, the density in the corona in our model is much higher and is closer to the density of the photosphere or the chromosphere. The rising magnetic flux tube has formed a low density region in its interior due to the higher magnetic pressure. When the tube then further rises to the corona, the density inside it is still lower than outside, because the coronal density is When the tube rise then further into the corona, the density inside the tube is still lower than outside, because the coronal density is rather high.

The ejection shown in the Figures 9 and 11 does not occur as a single eventit rather shows recurrent behavior. However, the periodicity is not as clear as in previous work (Warnecke and Brandenburg, 2010; Warnecke et al., 2011).

For Run A5, for example, we observe around 5 ejections during a time interval of about 1000 turnover times. A more clear indication for the recurrence of the ejections can be seen in Figure 12, where the normalized current density is averaged over two narrow latitude bands on each hemisphere.

\section{Conclusions}

In the present paper we have presented an extension of our two-layer approach by including a self-consistent convection zone into the model. We find a large-scale magnetic field generated by the convective turbulent motion in the convection zone. With the rotation, where the Coriolis number is larger than 3, we obtain a differential rotation pattern showing super-rotation, i.e. an equator rotating faster than the poles. The dynamo solutions we find are different and some of them have a periodic oscillatory behavior, where the large-scale magnetic field does not change sign; only the strength is varying. At the maxima, the velocity is suppressed due to the backreaction via the Lorentz force. Small-scale 

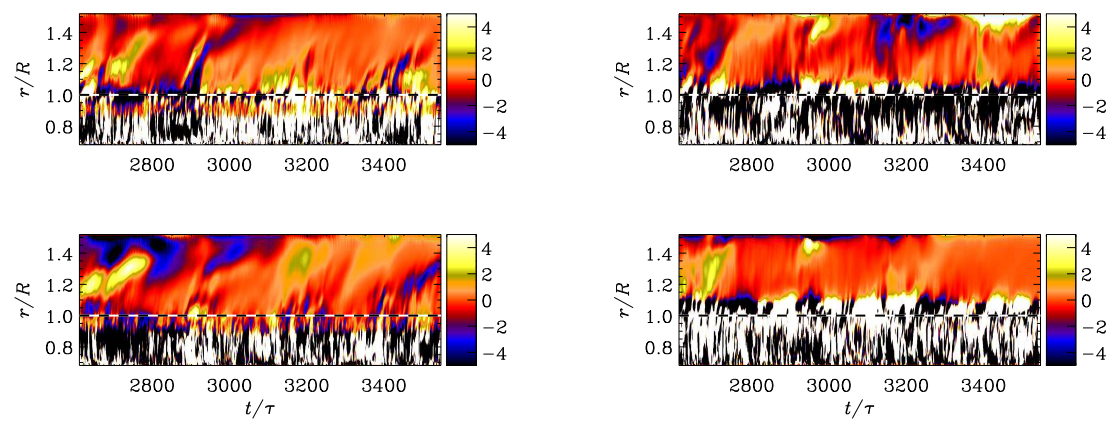

Figure 12. Dependence of the dimensionless ratio $\mu_{0} R \overline{\boldsymbol{J} \cdot \boldsymbol{B}} /\left\langle\overline{\boldsymbol{B}^{2}}\right\rangle_{t}$ on time $t / \tau$ and radius $r$ in terms of the solar radius. The top panels show a narrow band in $\theta$ in the northern hemisphere and the bottom ones in the southern hemisphere. We have also averaged in latitude from $4.1^{\circ}$ to $19.5^{\circ}$ (left panel) and $32.5^{\circ}$ to $45.5^{\circ}$ (right). Dark blue shades represent negative and light yellow positive values. The dotted horizontal lines show the location of the surface at $r=R$. Taken from Run A5

magnetic structures seem to show an equatorward migration near the equator and a poleward one near the poles.

Using a convectively-driven dynamo complicates the generation of ejections into the corona due to lower relative kinetic helicity. However, it was possible to produce ejections in two of the runs. The shape and the bipolar helicity structure is comparable with earlier work (Warnecke et al., 2011). Due to the high plasma $\beta$ in the corona, the ejections produce a local minimum of density which is carried along and ejected out of the domain. The ejections occur recurrently, but not clearly periodically, which is similar to the Sun.

An extension of the present work would include a detailed parameter study of cause and properties of the ejections. It includes also an advanced model for the solar corona with a lower plasma $\beta$ and a more efficient convection, which has a stronger stratification and is cooled by radiation. Another important aspect would be the generation of a self-consistent solar wind, which supports and interacts with the ejections.

Acknowledgements The authors are grateful for some discussion with Hardi Peter about the dynamics and rotation behavior of the solar corona. We acknowledge the allocation of computing resources provided by the Swedish National Allocations Committee at the Center for Parallel Computers at the Royal Institute of Technology in Stockholm and the National Supercomputer Centers in Linköping. Part of the computations have been carried out in the facilities hosted by the CSC - IT Center for Science in Espoo, Finland, who are financed by the Finnish ministry of education. This work was supported in part by the European Research Council under the AstroDyn Research Project No. 227952, the Swedish Research Council Grant No. 621-2007-4064, and the Academy of Finland grants 136189, 140970 (PJK) and 218159, 141017 (MJM). 


\section{References}

Antiochos, S. K., De Vore, C. R., Klimchuk, J. A.: 1999, ApJ 510, 485.

Archontis, V., Hood, A. W., Savcheva, A., Golub, L., DeLuca, E.: 2009, ApJ 691, 1276.

Brandenburg, A., Subramanian K.: 2005, Phys. Rep. 417, 1-209.

Blackman, E. G., Brandenburg, A.: 2003, ApJ 584, L99.

Brun, A. S., Miesch, M. S., Toomre, J.: 2004, ApJ 614, 1073.

Cantiello, M., Braithwaite, J., Brandenburg, A, Del Sordo, F., Käpylä, P. J., Langer, N.: 2011, IAU Symposium 272, 32.

Cantiello, M., Braithwaite, J., Brandenburg, A, Del Sordo, F., Käpylä, P. J., Langer, N.: 2011, IAU Symposium 273, 200.

Elliot, J. R., Miesch, M. S., Toomre, J.: 2000, ApJ 533, 546.

Fang, F., Manchester, W., Abbett, W. P., van der Holst, B.: 2010, ApJ 714, 1649.

Käpylä, P. J., Korpi, M. J., Brandenburg, A.: 2008, A\&GA 491, 353.

Käpylä, P. J., Korpi, M. J., Brandenburg, A., Mitra, D., Tavakol, R.: 2010, AN 331, 73.

Käpylä P. J.,Korpi, M. J., Guerrero, G., Brandenburg, A.,Chatterjee, P.: 2011a, A 6 A 531, A162.

Käpylä, P. J., Mantere, M. J.,Brandenburg, A.: 2011b, http://arxiv.org/abs/1109.4625

Levine, R. H., Schulz, M., Frazier, E. N.: 1982, Solar Phys. 77, 363.

Low, B. C.: 1996, Solar Phys. 167, 217.

Martnez-Sykora, J., Hansteen, V., Carlsson, M.: 2008, ApJ 679, 871.

Mitra, D., Tavakol, R., Brandenburg, A., Moss, D.: 2009, ApJ 697, 923.

Miesch, M. S., Elliot, J. R., Toomre, J. Clune, T. L., Glatzmaier, G. A. Gilman P. A.: 2000, ApJ 532, 59.

Ossendrijver, M., Stix, M., Brandenburg, A.: 2001, A\&A 376713.

Plunkett, S. P., Vourlidas, A., Šimberová, S., Karlický, M., Kotrč, P., Heinzel, P., Kupryakov, Y. A., Guo, W. P., Wu, S. T.: 2000, Solar Phys. 194, 371.

Régnier, S., Amari, T., Kersalé, E.: 2002, A\&A 392, 1119.

Roussev, I. I., Forbes, T. G., Gombosi, T. I., Sokolov, I. V., DeZeeuw, D. L., Birn, J.: 2002, ApJ 588, L45.

Sturrock, P. A.: 1980, Solar Flares Colorado Associated University Press, Boulder.

Thompson, W. T. , Kliem, B., Török, T.: 2011, Solar Phys. DOI: 10.1007/s11207-0119868-5.

Török, T., Kliem, B.: 2003, A\&GA 406, 1043.

Timothy, A. F., Krieger, A. S., Vaiana, G. S.: 1975, Solar Phys. 42, 135.

Vainshtein, S. I. and Cattaneo, F.: 1992, Apj 393, 165.

Warnecke, J., Brandenburg, A.: 2010, A\&A 523, A19.

Warnecke, J., Brandenburg, A., Mitra, D.: 2011, A\&A 534, A11.

Wöhl, H., Brajša, R., Hanslmeier, A., Gissot, S. F.: 2010, A\&A 520, A29. 
SOLA: sol_paper.tex; 5 December 2011; $1: 17$; p. 18 